\title{
Cerilla, mechero, fuego: claves de recuperación y recuerdo infantil
}

\author{
CARMEN ORTí \\ GISÈLE MARTY
}

Universidad Complutense Madrid y Universidad de las Islas Baleares

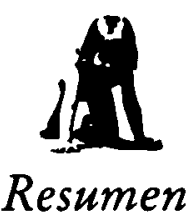

Esta investigación tiene como objeto averiguar el efecto de claves visuales que representan diversas relaciones específicas entre objetos, en pruebas de memoria aplicadas a 611 niños de siete años de edad, divididos en seis grupos experimentales. Los resultados sugieren un mejor recuerdo de las asociaciones sintagmáticas frente a las asociaciones paradigmáticas. Este becho puede interpretarse como un dominio de la memoria episódica sobre la semántica en una determinada etapa evolutiva (aunque una interpretación alternativa sugiere que la interacción entre tarea y prueba tiene más peso que la interacción entre tarea y sujeto). Si se acepta el dominio de la memoria episódica en una cierta etapa evolutiva, queda pendiente de averiguar si este dominio se mantiene o no a lo largo de etapas posteriores $y$, en caso afirmativo, cuáles serian las implicaciones.

Palabras clave: Recuerdo con clave, Memoria infantil, Niveles de procesamiento.

\section{Match, lighter, fire: Retrieval cues and child recall}

\section{Abstract}

The effect of certain visual cues, representing specific relations between objects, in memory tests given to 611 7-yr olds divided into six experimental groups was the object of the present study. Results suggest better recall of syntagmatic than paradigmatic associations. This may be interpreted in terms of episodic memory being more dominant than semantic memory at certain stages of development (although an alternative interpretation suggests that interaction between the orientation task and criterion task is more important than interaction between the former and the subject). If this dominance of episodic memory at a certain developmental stage is accepted, then it remains to be investigated whether this is maintained through subsequent stages of development; and if this is the case, its possible implications.

Keywords: Cued recall, Developmental memory, Levels of processing.

Dirección de la autora: Universidad Complutense de Madrid. Facultad de Psicología. 28023 Madrid.

Original recibido: Julio, 1988. Revisión recibida: Octubre, 1989. Aceptado: Noviembre 1989. 


\section{INTRODUCCION}

Este trabajo pretende investigar el efecto de determinadas claves visuales sobre el reconocimiento y el recuerdo de objeto por los niños, examinando los aspectos diferenciales que aparecen en la recuperación de la información almacenada cuando en el procesamiento de dicha información se han establecido distintas relaciones entre los objetos.

El punto de partida metodológico se sitúa en el enfoque de los niveles de procesamiento. Como es conocido de todos, esta hipótesis supone que a mayor complejidad o "profundidad" de procesamiento existe un mayor recuerdo (Craik y Lockhart, 1972). El método experimental utilizado para investigar la naturaleza de los procesos cognitivos en el enfoque de los niveles de procesamiento, consiste en el procedimiento de aprendizaje incidental con tareas de orientación. Craik y Tulving (1975) utilizaron inicialmente las tareas de orientación para inducir tanto procesos no semánticos como procesos semánticos. Sin embargo, la dificultad para separar «operaciones de orientación semántica» y "no semántica», nos ha llevado a establecer solamente tareas de orientación semántica. Dentro de esta orientación, las tareas propuestas pretenden favorecer un tipo de procesamiento frente a otros posibles, de acuerdo con la noción general de dominios de procesamiento y códigos focales (Nelson, 1979; Baddeley, 1976). Según esta teoría, se codifican atributos de todos los niveles, pero las demandas de la situación hacen que ciertas características resulten más sobresalientes y se codifiquen con mayor intensidad.

Tal como indican Aparicio y Zacagnini (1980), las tareas de orientación serán válidas mientras no se fuerce su utilización y no se exija más de lo que pueden ofrecer, es decir, mientras se oriente en una situación neutra el tipo de procesamiento que hará el sujeto.

En la presente investigación se incluye el uso de claves para la recuperación de la información, hecho que suéle relacionarse con los presupuestos teóricos ligados al concepto de memoria episódica. El concepto de memoria episódica aqui defendido se acerca al concepto de "memoria en sentido estricto" de Piaget (Piaget e Inhelder, 1968), y nos ha parecido sugerente orientar nuestro trabajo en la línea piagetiana, ya que los sujetos a los que se ha dirigido esta experiencia, se encuentran en una etapa especialmente interesante: al final del subperiodo preoperacional o principio del subperíodo de las operaciones concretas (Teniendo en cuenta que la mayoría de los trabajos realizados utilizan estímulos verbales presentados a sujetos adultos, hemos optado, siguiendo una idea de Rivière, por un experimento en el que sean niños los sujetos que deben clasificar dibujos).

Sabemos que en todos los niveles genéticos piagetianos la cognición puede caracterizarse en función del tipo de acciones que realiza el sujeto. Durante el período sensorio-motor las acciones están externalizadas, pero a medida que el niño se aleja de los años infantiles estas acciones se internalizan y «esquematizan", despojándose paulatinamente de sus cualidades concretas.

La cuestión fundamental que plantea Piaget es la comparación entre la memoria "en sentido estricto" y la conservación de los esquemas, y a tal efecto sostiene que la memoria «en sentido estricto» constituye el aspecto figurativo de la conservación de los esquemas. Las mejores imágenes para 


\section{3}

un esquema de pensamiento son las que representan las situaciones en las cuales estuvo en acción y, por así decirlo, donde se concretó. Cuanto más estrecha aparece esta relación entre los elementos operativos y figurativos de la memoria, más tiende a desaparecer el carácter propio de la memoria «en sentido estricto".

A partir de estas premisas, nos planteamos una serie de interrogantes que intentamos despejar sometiendo nuestros grupos experimentales a diferentes tareas relacionales y analizando sus efectos en distintas pruebas de memoria.

Teniendo presente que la memorización y el recuerdo están sujetos a un conjunto de determinantes simultáneos, aplicamos el modelo tetraédico de Jenkins (1979) al analizar las principales variables de nuestros experimentos. Dicho modelo ofrece un tipo de esquema clasificatorio que recoge los materiales, las estrategias o tareas, las pruebas o tareas-criterio y las características de los sujetos, junto con las interacciones entre dichas variables.

\section{METODOLOGIA}

La metodología general de nuestros experimentos se expone a continuación, al especificar las cuatro categorías de variables:

\section{El material}

- Hoja de Estímulos: Estímulos visuales diseñados por las autoras consistentes en doce dibujos de objetos comunes, iguales para todos los sujetos (ej.: caja de cerillas). A cada uno de los dibujos le corresponde un número que permite establecer relaciones con los dibujos de las hojas de respuesta.

- Hoja de Respuesta: Tres tipos de hoja de respuesta consistente cada una en una serie de doce dibujos relacionables con los estímulos y distintos para cada condición de relación (por ejemplo para el estímulo "caja de cerillas» se ha asociado en la relación formal: un prisma recto; en la relación paradigmática: un mechero; en la relación sintagmática: unos leños ardiendo).

- Hoja de Reconocimiento: contiene 40 dibujos.

\section{Las tareas}

Se utilizan tres tipos de tareas definidas por la condición de relación que se establece durante la fase de aprendizaje:

- Relación de tipo formal: asociar un objeto con su forma. se.

- Relación de tipo paradigmático: asociar dos objetos de la misma cla-

- Relación de tipo sintagmático: asociar el objeto-estímulo con el dibujo de la hoja de respuesta que tenga más relación.

Se comprueba que las tareas presentan un mismo grado de dificultad. 


\section{4}

3. Las pruebas:

Se utilizan tres tipos de pruebas:

A) pruebas de aprendizaje: una para cada tipo de tarea.

B) pruebas de reconocimiento: a las 24 horas y a las 48 horas, para los tres tipos de tareas.

C) pruebas de recuerdo con clave a las 24 horas y a las 48 horas para los tres tipos de tarea. En el segundo experimento, pruebas de recuerdo con clave a las 24,48 y 72 horas para las tareas de relación sintagmática y de relación paradigmática.

En cada prueba se evalúan los aciertos con un punto positivo. Los errores y falsos reconocimientos se anotan (por su posible utilidad en otras investigaciones), pero no se contabilizan.

\section{Los sujetos:}

Los sujetos son 611 niños de ambos sexos, con una edad media de 7 años, alumnos de $2 .^{\circ}$ de E.G.B.

Nos interesa ahora preguntarnos qué tipo de relación recordarán mejor los niños, teniendo en cuenta que las relaciones sintagmáticas implican algún tipo de sucesión o movimiento en el tiempo o en el espacio, y que son relaciones que pueden recordar más fácilmente alguna actividad concreta, algún episodio de la propia vida del niño y que, por otra parte, las relaciones paradigmáticas implican un parecido familiar y son más propias de la llamada memoria «en sentido amplio" o memoria semántica.

\section{Experimento I}

La primera fase de esta experiencia se llevó a cabo en tres etapas:

1) Aprendizaje.

2) Reconocimiento.

3) Recuerdo con clave de cada una de las condiciones citadas anteriormente (formal, paradigmática y sintagmática).

- Material. El material utilizado en cada etapa fue el siguiente:

1) Aprendizaje:

- Una hoja de estímulos conteniendo doce estímulos visuales (los mismos para todos los sujetos).

- Una hoja de respuesta conteniendo doce dibujos que el sujeto debía relacionar con los estímulos anteriores, estableciendo una asociación de tipo formal, sintagmático o paradigmático, según al grupo al que estuviera asignado.

2) Reconocimiento:

- Hoja de reconocimiento con 40 dibujos, en la que habían de reconocer los doce estímulos que vieron en la sesión anterior.

3) Recuerdo con clave:

- Hoja de respuesta, en la que habían de escribir junto a cada dibujo, el nombre del objeto con el que lo habían relacionado el día anterior. 
- Sujetos. Se utilizó una muestra de 163 sujetos, (85 niños y 78 niñas), de edad media 7 años, estudiantes de 2..$^{\circ}$ de E.G.B. en la Escuela Pública de Prácticas (2 grupos) y en el Colegio Nacional Eugenio López (4 grupos) de Palma de Mallorca.

- Tiempo. Libre.

- Procedimiento. La aplicación de las pruebas se realizó en dos sesiones; en la primera de ellas el Aprendizaje, y en la segunda el Reconocimiento y el Recuerdo con clave.

Todas las pruebas fueron aplicadas de forma colectiva, en el aula habitual de cada grupo.

El intervalo de tiempo entre la primera y la segunda sesión fue de 24 horas para 3 grupos (uno por cada condición experimental) y de $\mathbf{4 8}$ horas para los 3 restantes.

En la primera sesión se les repartió una hoja de respuesta boca abajo en la que habían de escribir sus datos personales, y una hoja de estímulos también boca abajo, en la que no debían escribir nada.

Una vez puestos los datos personales, se leían las instrucciones correspondientes a la condición con que se trabajaba en ese momento, sin advertir el tiempo de duración, ni la existencia de pruebas posteriores.

En la segunda sesión se les entregó una hoja de reconocimiento, en la que habían de encontrar entre 40 dibujos, los 12 estímulos que vieron en la sesión anterior.

Posteriormente, después de recoger las hojas de reconocimiento, se volvían a repartir hojas de respuesta boca abajo, que contenían los dibujos correspondientes a la condición (formal, sintagmática o paradigmática) con la que habían trabajado en la primera sesión, y que serían las claves para el recuerdo pidiéndoles que recordaran el objeto con el que habían relacionado cada dibujo en la sesión anterior.

- Evaluación. Se evaluaron los aciertos con un punto positivo en todos los casos (aprendizaje, reconocimiento, recuerdo con clave); los errores y falsos reconocimientos fueron anotados (por su posible utilidad en otras investigaciones), pero no se contabilizaron.

- Resultados y discusión. Una vez efectuado el estudio estadístico correspondiente (prueba de comparación de dos medias observadas en muestras pequeñas con datos independientes) encontramos (Tabla I):

a) En.las tareas de Aprendizaje, como era de esperar, no aparecieron diferencias estadísticamente significativas en ninguno de los seis grupos (N. C. $95 \%$ ).

b) En las tareas de Reconocimiento, tampoco aparecieron diferencias estadísticamente significativas en ninguno de los seis grupos (N. C. $95 \%$ ).

c) En el Recuerdo con clave de la condición Formal (N. C. $95 \%$ ), no encontramos diferencias estadísticamente significativas entre los dos intervalos de tiempo ( 24 y 48 horas), ni con el resto de los grupos.

En el Recuerdo con clave de la condición Paradigmática encontramos una diferencia estadísticamente significativa (N. C. $95 \%$ ) entre ambos intervalos de tiempo. 


\section{6}

TABLA I

Experimento I

\begin{tabular}{|c|c|c|c|c|c|c|c|c|c|c|c|c|}
\hline & \multicolumn{6}{|c|}{ Intervalo 24 horas } & \multicolumn{6}{|c|}{ Intervalo 48 horas } \\
\hline & \multicolumn{2}{|c|}{ Aprendiz } & \multicolumn{2}{|c|}{ Reconoc. } & \multicolumn{2}{|c|}{ R. clave } & \multicolumn{2}{|c|}{ Aprendiz } & \multicolumn{2}{|c|}{ Reconoc. } & \multicolumn{2}{|c|}{ R. clave } \\
\hline & $\overline{\mathbf{x}}$ & o & $\overline{\mathbf{x}}$ & $\mathbf{o}$ & $\overline{\mathbf{x}}$ & $\sigma$ & $\overline{\mathbf{x}}$ & $\sigma$ & $\overline{\mathbf{x}}$ & $\sigma$ & $\overline{\mathbf{x}}$ & $\sigma$ \\
\hline $\begin{array}{l}\text { R.F. } \\
\text { R.P. } \\
\text { R.S. }\end{array}$ & $\begin{array}{c}11,68 \\
11,5 \\
11,6\end{array}$ & $\begin{array}{l}0,69 \\
1 \\
0,76\end{array}$ & $\begin{array}{l}10,93 \\
10,45 \\
10,43\end{array}$ & $\begin{array}{l}1,11 \\
1,47 \\
1,35\end{array}$ & $\begin{array}{c}11,13 \\
9,66 \\
9,6\end{array}$ & $\begin{array}{l}1,81 \\
1,69\end{array}$ & $\begin{array}{l}11,68 \\
11,33 \\
11,43\end{array}$ & $\begin{array}{l}0,54 \\
0,92 \\
0,97\end{array}$ & $\begin{array}{r}10,17 \\
9,93 \\
9,52\end{array}$ & $\begin{array}{l}1,31 \\
1,62 \\
1,24\end{array}$ & $\begin{array}{r}11,03 \\
5,16 \\
8,59\end{array}$ & $\begin{array}{l}4,27 \\
1,94\end{array}$ \\
\hline
\end{tabular}

En el Recuerdo con clave de la condición Sintagmática no encontramos diferencias estadísticamente significativas (N. C. $95 \%$ ) entre ambos intervalos de tiempo.

También encontramos una diferencia estadísticamente significativa (N.C. $95 \%$ ), al comparar el Recuerdo con Clave de la condición sintagmática-48 horas y el Recuerdo con Clave de la condición paradigmática-48 horas.

La interpretación de estos resultados nos indica que el Recuerdo con Clave disminuye significativamente a lo largo del tiempo en los sujetos que tuvieron que recordar objetos de una misma clase (como cerillas-mechero), pero no en los sujetos que recordaron una relación de tipo sintagmático (cerillas-fuego).

Es decir, que el niño recuerda mejor relaciones que evocan alguna actividad conocida, algún episodio de su propia vida; en pocas palabras, relaciones que pertenecen a la llamada memoria episódica o memoria «en sentido estricto" si utilizamos la terminología de Piaget.

De todos es sabido que, de una forma semejante a la distinción que haría posteriormente Tulving entre memoria episódica y memoria semántica, Piaget tiene en cuenta dos tipos de memoria diferentes: la memoria en sentido estricto, que corresponde a la "recognición de objetos», de secuencias, de acontecimientos concretos, y tiene por tanto una referencia explícita al pasado, y la memoria en sentido amplio, que engloba la capacidad del sujeto de reproducir lo generalizable, es decir el "esquematismo".

Tal y como señala el propio Piaget, la primera diferencia entre la memoria en sentido estricto y la conservación de los esquemas consiste en que éstos se actualizan en la situación presente sin referencia explícita al pasado.

El concepto de memoria episódica conlleva el principio de especificidad de codificación, y por esta razón, la metodología empleada en nuestro trabajo permite que en las condiciones experimentales propuestas, se cumpla el mencionado principio, ya que en todas ellas las claves de recuperación se han almacenado como huellas concretas de memoria para estos acontecimientos. Sin embargo, y de acuerdo con las críticas formuladas por Baddeley (1976) y Diges y Seoane (1981) a la distinción entre memoria episódica-memoria semántica (Tulving, 1972) y al principio de «especificidad de codificación" (Tulving y Thomson, 1973), en esta experiencia no nos ha interesado poner de relieve la eficacia de las claves de recuperación en función de su capacidad de reinstaurar la forma exacta en que la información 
fue codificada durante la fase de aprendizaje. Lo que hemos pretendido es saber por qué algunos tipos de clave consiguen mejores resultados que otros. De hecho, hemos de señalar, que el propio Tulving (1983) flexibilizó de manera considerable el principio de especificidad de codificación. Por tanto, no concebimos aquí la memoria episódica como un almacén independiente.

El interrogante que ahora nos planteamos es el siguiente: ¿qué implica una relación sintagmática y por qué los niños la recuerdan mejor en distintos intervalos de tiempo?

La respuesta que nos parece adecuada es la de que una relación más dinámica podría favorecer, por parte de los niños, la construcción a nivel de aprendizaje de un acontecimiento propio vivido, que facilitaría el recuerdo posterior. Si esto es así, el mejor recuerdo de la condición sintagmática tendría que mantenerse en los distintos intervalos de tiempo, como hemos verificado en la presente investigación.

\section{Experimento II}

Animados por estos resultados, decidimos continuar la investigación con una muestra más numerosa y bajo las siguientes premisas:

- Trabajar con las condiciones sintagmática y paradigmática, ya que en la relación formal no hallamos diferencias dignas de mención.

- En dos etapas (Aprendizaje y Recuerdo con Clave), suprimiendo la etapa de Reconocimiento, en la que no encontramos diferencias estadísticamente significativas en ningún caso. 72 horas).

- Con tres intervalos de tiempo diferentes entre ambas fases $(24,48 \mathrm{y}$

- Material: El material utilizado en cada etapa fue el siguiente:

Aprendizaje (una hoja de estímulos conteniendo doce estímulos visuales, los mismos para todos los sujetos, y una hoja de respuesta para cada condición, en la que el sujeto debería establecer la relación correspondiente según el grupo al que estuviera asignado).

Recuerdo con Clave (una hoja de respuesta para cada sujeto, correspondiente a la condición experimental en que hizo el Aprendizaje).

- Sujetos: La muestra definitiva de la presente experiencia fue de 448 sujetos de ambos sexos (234 niños y 214 niñas), edad media 7 años, alumnos de 2..$^{\circ}$ de EGB, distribuidos en los siguientes grupos y Centros Mixtos de Enseñanza:

\section{Condición Sintagmática}

(24 h.) 77 sujetos, Colegio Público de Calviá

(48 h.) 88 sujetos, Colegio CIDE

(72 h.) 82 sujetos, Colegios Nacionales de El Arenal

Condición Paradigmática

(24 h.) 64 sujetos, Colegio Gabriel Valseca

(48 h.) 64 sujetos, Colegio CIDE

(72 h.) 73 sujetos, Colegio Son Rullán, El Arenal

-Tiempo: libre.

-Procedimiento: La aplicación de las pruebas se realizó en dos sesio- 


\section{8}

nes; en la primera de ellas el Aprendizaje, y en la segunda el Recuerdo con Clave.

Todas las pruebas fueron aplicadas de forma colectiva en el aula habitual de cada grupo.

El intervalo de tiempo entre la primera y la segunda sesión fue de 24, 48 ó 72 horas, según las condiciones a que fueron asignados los grupos.

En la primera sesión se les repartió una hoja de respuesta boca abajo en la que habían de escribir sus datos personales, y una hoja de estímulos también boca abajo en la que no debían escribir nada.

Una vez puestos los datos personales, se leían las instrucciones correspondientes a la condición con que se trabajaba en ese momento, sin advertir el tiempo de duración ni la existencia de pruebas posteriores.

En la segunda sesión se repartió de nuevo a los sujetos una hoja de respuesta, que contenía los dibujos correspondientes a la condición (sintagmática o paradigmática) con la que habían trabajado en la etapa de aprendizaje, pidiéndoles que recordaran el nombre del objeto con el que relacionaron cada dibujo el día anterior.

- Evaluación: Se evaluaron con un punto positivo los aciertos, tanto en el Aprendizaje como en el Recuerdo con Clave. Los errores fueron anotados (por su posible utilidad en otras investigaciones), pero no se contabilizaron.

- Resultados y discusión: Los resultados del estudio estadístico realizado (prueba de comparación de dos medias estudiadas en muestras grandes con datos independientes) indican (Tabla II):

TABLA II

Experimento II

Recuerdo con clave

\begin{tabular}{lcccccc}
\hline & \multicolumn{2}{c}{ Intervalo 24 horas } & \multicolumn{2}{c}{ Intervalo 48 horas } & \multicolumn{2}{c}{ Intervalo 72 horas } \\
\cline { 2 - 7 } & $\overline{\mathbf{x}}$ & $\sigma$ & $\overline{\mathbf{x}}$ & $\sigma$ & $\overline{\mathbf{x}}$ & $\sigma$ \\
\hline R.P. & 9,40 & 16,23 & 5,21 & 14,43 & 4,87 & 9,65 \\
R.S. & 9,65 & 16,73 & 9,51 & 11,94 & 9,35 & 17,93 \\
\hline
\end{tabular}

a) Una diferencia estadísticamente significativa (N.C. $95 \%$ ) en el Recuerdo con Clave de la condición paradigmática entre los intervalos 24 horas-72 horas.

b) En el Recuerdo con Clave de la condición sintagmática, no aparecen diferencias estadísticamente significativas entre los diferentes intervalos de tiempo.

c) También resulta estadísticamente significativa (N.C. $95 \%$ ) la diferencia hallada entre el Recuerdo con Clave de ambas condiciones, C. Sintagmática-48 horas y C. Paradigmática-48 horas.

d) En el Recuerdo con Clave de ambas condiciones, intervalo 72 horas, también aparecen diferencias estadísticamente significativas.

e) En la tarea de Aprendizaje no existen diferencias estadísticamente significativas (N.C. $95 \%$ ) en ninguno de los grupos. 
Estos resultados corroboran los anteriores obtenidos en nuestra primera experiencia, en el sentido de que el Recuerdo con Clave disminuye significativamente a lo largo del tiempo en sujetos que tuvieron que recordar objetos de la misma clase (cerillas-mechero, paraguas-chubasquero, balónpelota de tenis), pero no en los sujetos que hubieron de recordar relaciones de tipo sintagmático (cerillas-fuego, paraguas-lluvia, balón-portería de fútbol).

Aunque somos conscientes de que los análisis estadísticos realizados en esta ocasión son insuficientes y consideramos que el análisis factorial de la varianza aportaría una mayor consistencia a nuestros resultados, hemos optado por mantener la comparación de dos medias observadas en muestras pequeñas ya que estos experimentos fueron el primer paso de una línea de investigación más amplia actualmente en curso y en la que se están llevando a cabo análisis estadísticos más profundos.

\section{DISCUSION Y CONCLUSIONES}

Enmarcaremos nuestra discusión, tal y como lo sugiere el modelo de Jenkins (1979), en el análisis de las interacciones más relevantes que existen entre las principales variables de nuestros experimentos.

\section{Interacción entre tarea y prueba}

Al ser nuestro punto de partida metodológico el enfoque de los niveles de procesamiento, este tipo de interacción es de especial importancia. De los distintos tipos de tareas y pruebas utilizadas, resultaron significativas las diferencias en recuerdo con clave de la tarea de relación paradigmática en distintos intervalos de tiempo y en comparación con la tarea de relación sintagmática. Es decir, nuestros resultados muestran un recuerdo significativamente mejor de las relaciones de tipo sintagmático.

Mientras que las relaciones de tipo sintagmático establecen vínculos entre elementos particulares, las relaciones de naturaleza paradigmática se establecen entre clases de elementos, teniendo un carácter más conceptual. $\mathrm{Al}$ tratarse de una prueba de recuerdo que exige el manejo de las relaciones entre elementos particulares, las relaciones de tipo sintagmático van asociadas a mejores resultados de recuerdo. Por tanto la interacción entre la tarea y la prueba parece ser un factor importante en la interpretación de nuestros resultados.

\section{Interacción entre sujeto y material}

Las pruebas de aprendizaje demostraron que las estructuras relacionales del material son apropiadas a los conocimientos de los sujetos. En cuanto a la interacción entre tarea y material, la mayoría de los trabajos utilizan palabras como material de una situación particular de aprendizaje-memoria o estudian las diferencias entre palabras e imágenes. En estos experimentos, se ha utilizado un material visual y sería interesante averiguar si se obtiene el mismo tipo de resultados con un material verbal. 
Interacción entre sujeto y tarea

Tiene una importancia especial para la psicología evolutiva. Los autores de este campo nos incitan a pensar en el sistema de memoria como un sistema en constante evolución y desarrollo, y a considerar la propia adquisición del conocimiento como una forma de memoria. Es dentro de esta perspectiva evolutiva donde aparencen las explicaciones más interesantes acerca de por qué los niños recuerdan mejor las relaciones sintagmáticas.

Ya hemos sugerido que las relaciones sintagmáticas pueden evocar más fácilmente alguna actividad concreta o algún acontecimiento de la propia vida del niño, es decir, que pertenecen a la llamada memoria «episódica» o memoria «en sentido estricto» si utilizamos la terminología de Piaget.

Nos ha parecido interesante situar esta posible interpretación de nuestros datos en el marco de las etapas piagetianas teniendo en cuenta sobre todo que el planteamiento de la escuela de Ginebra es considerado por todos los autores preocupados por la perspectiva evolutiva del estudio de la memoria como uno de los enfoques más importantes, quizá el más importante de todos (Marchesi, 1984; Gregg, 1986). Además, hay que recordar que la perspectiva evolutiva tiene hoy en día una especial relevancia como sano contrapunto al enfoque del procesamiento de la información que ofrece la imagen de un sujeto estático (Riviere, 1987).

De los dos tipos de memoria que Piaget tiene en cuenta, la memoria «en sentido estricto» corresponde a la recognición de objetos, secuencias y acontecimientos concretos, y tiene por tanto una referencia explícita al pasado, mientras que la memoria «en sentido amplio» engloba la capacidad del sujeto de reproducir lo generalizable, es decir «el esquematismo». Al tener un carácter más conceptual, las relaciones paradigmáticas pertenecen sobre todo a la memoria «en sentido amplio». Por su parte, las relaciones sintagmáticas, más dinámicas, podrían favorecer por parte de los niños la construcción, a nivel de aprendizaje, de un acontecimiento propio vivido que facilitaría el recuerdo posterior. Si esto es así, el mejor recuerdo de la condición sintagmática tendría que mantenerse en los distintos intervalos de tiempo, tal como hemos verificado en la presente investigación.

Este mejor recuerdo de tipo episódico nos lleva a postular un predominio de la memoria episódica sobre la semántica en los niños cuyos esquemas están en formación y por tanto, tal y como indican Piaget e Inhelder (1968), son más dependientes de los aspectos espacio-temporales en los cuales se sitúan las relaciones de movimiento.

A lo largo de su obra, Piaget demuestra que en el terreno de la memoria se hallan etapas sucesivas que pueden fácilmente situarse en el cuadro de los niveles de desarrollo de la inteligencia. La etapa de las recogniciones, que corresponde al desarrollo sensoriomotor; la de las reconstituciones, que señala el paso del nivel sensoriomotor al representativo; y la de las evocaciones, que corresponde al nivel de las formas representativas preoperatorias u operatorias de la inteligencia. Es interesante preguntarse si el mejor recuerdo de las relaciones sintagmáticas se mantiene en las distintas etapas o si, por el contrario, desaparece en los últimos niveles. De este modo aparecen varias hipótesis que pueden ser útiles para investigaciones posteriores, dentro del mismo horizonte teórico piagetiano que hemos utilizado.

Sin embargo, no podemos dejar de mencionar que se suele reprochar a 
Piaget el haber ofrecido una imagen del desarrollo cognitivo demasiado lógico-formal, imagen en la cual el sujeto de las últimas etapas no tiene contexto propio. Pero si el mejor recuerdo de las relaciones sintagmáticas se mantuviera en estas últimas etapas, entonces se podría volver a plantear una serie de preguntas sobre las características, el papel y la evolución de la llamada memoria en sentido estricto o memoria episódica.

Una interpretación alternativa del mejor recuerdo de las relaciones sintagmáticas (ya sugerida anteriormente), da más peso a la interacción entre sujeto y prueba que a la interacción entre tarea y sujeto, al poner de relieve que, en la medida en que las relaciones sintagmáticas implican la existencia de vínculos más particulares entre los estímulos que las relaciones paradigmáticas, favorecen también en mayor grado una conexión más individualizada (más "específica») entre la clave y la diana. Esta última interpretación se podría ver confirmada también si se mantuviera un mejor recuerdo de las relaciones sintagmáticas con sujetos de otras etapas y/o con otro tipo de material.

Concluiremos con Jenkins (1979) subrayando que las interacciones abundan y que las generalizaciones claras van a ser difíciles de establecer. Por el momento sólo podemos afirmar de una manera terminante que:

«Si los sujetos son niños de 7 años que cursan 2. de EGB y si las tareas requieren relaciones sintagmáticas (más que paradigmáticas) y si el material consta de simples dibujos de objetos y si la prueba es de recuerdo con clave, entonces se observará un nivel relativamente alto de recuerdo que se mantendrá en distintos intervalos de tiempo.»

Este tipo de conclusión abre la puerta a muchos posibles experimentos, algunos de los cuales han sido sugeridos en esta discusión.

\section{Referencias}

APARICIO, J. J., y ZACCAGNINI, J. L. (1980). «¿Qué se recuerda cuando no se recuerda? Niveles de procesamiento". Revista de Psicología General y Aplicada, 35, pp. 901-912.

Baddeley, A. D. (1976). The Psychology of Memory. Nueva York, Basic Books.

CRAIK, F. I. M., y LOCKHART, R. S. (1972). «Levels of processing: A framework for memory research». Journal of Verbal Learning and Verbal Bebavior, 11, pp. 671-684.

Craik, F. I. M., y Tulving, E. (1975). "Depth of processing and the retention of words in episodic memory*. Joumal of Experimental Psychology, 104, Pp. 268-294.

DigES, M., y SEOANE, J. (1981). "Análisis experimental de la memoria: estudios episódicos y semánticos». Ponencia presentada en la Reunión Internacional sobre Psicología y procesos de socialización. Alicante.

GREGG, V. H. (1986). Introduction to human memory. Londres: Routledge \& Kegan Paul.

JENKINS, J. J. (1979). «Four points to remember: A Tetrahedral Model of Memory Experiments». En Cermak y Craik (eds.): Levels of Processing in Human Memory, Hillsdale: NJ, LEA.

MarCHeSI, A. (1984). «El desarrollo de la memoria». En J. Palacios, A. Marchesi y M. Carretero (eds.): Psicología evolutiva II: Desarrollo cognitivo y social del niño, Madrid: Alianza.

NELSON, D. L. (1979). «Remembering pictures and words: appearance, significance and name». En Cermak y Craik (eds.): Levels of Processing in Human Memory, Hillsdale: NJ, LEA.

PIAGet, J., e INHelder, B. (1968). Mémoire et intelligence. Paris: P.U.F.

RIVIERE, A. (1987). El sujeto de la Psicologia Cognitiva. Madrid: Alianza.

TULVING, E. (1972). «Episodic and semantic memory*. En E. Tulving y W. Donaldson (eds.): Organisation and memory. Nueva York: Academic Press.

- (1983). Elements of Episodic Memory. Oxford: Oxford University Press.

Tulving, E., y THOMSON, D. M. (1973). «Encoding specifity and retrieval processes in episodic memory». Psychological Review, 80, pp. 352-373. 


\section{Extended summary}

This study sought to research the effect of certain visual cues on child recognition and memory of objects, examining the differential aspects appearing in the retrieval of stored information, when during the processing of this information certain relations have been established.

A series of questions were raised which we attempted to answer by subjecting our experimental groups to different relational tasks, and analysing their effects in different memory tests.

Visual stimuli used in the study consisted in twelve pictures of everyday objects, associated with other pictures, corresponding to each of the relationships designed: a formal relation, in wich an object is associated with its form (matchbox-straight prism); a paradigmatic relation, in which two objects of the same class are associated (matchbox-lighter); and a syntagmatic relation, in which the object-stimuli is associated with another object with whom it has some type of relation (match box-burning logs).

Three types of tests were carried out: Learning (one for each type of task); recognition (at 24 and 48 hours for the three different task); and cued recall (at 24 and 48 hours for the three different tasks in the first experiment, and at 24, 48, and 72 hours for syntagmatic and paradigmatic relations in the second experimented).

The sample consisted of 611 primary school boys and girls in their second year with an average age of 7 yrs.

One of the aims of the study was to determine which type of relation the children remembered best - syntagmatic or, paradigmatic - taking into account that the first involves some type of succession in time or space, and also that it is a relationship which is easier to associate with some specific activity or previous event in the child's life; the second type, however, involves a familiar likeness and it is closer to semantic memory.

The results obtained suggest better recall of syntagmatic associations, which may be interpreted as a dominance of syntagmatic over paradigmatic memory at a certain developmental stage. If this is accepted, the next step would be to find out if this dominance is maintained throughout subsequent stages of development; and if this were the case, to study its possible implications. 